\title{
Article \\ Communication Bandwidth Prediction Technology for Smart Power Distribution Business in Smart Parks
}

\author{
Xia Zhou ${ }^{1, *}$, Jianqiang Lu ${ }^{1}$, Xiangpeng Xie ${ }^{1}$, Chengjie Bu ${ }^{1}$, Lei Wan ${ }^{1}$ and Feng Xue ${ }^{2}$ \\ 1 Institute of Advanced Technology, Nanjing University of Post and Telecommunication, \\ Nanjing 210032, China; ljq109811@163.com (J.L.); xiexp@njupt.edu.cn (X.X.); buchengjie1@163.com (C.B.); \\ wanlei232812@163.com (L.W.) \\ 2 NARI Group Corporation, State Grid Electric Power Research Institute, Nanjing 211106, China; \\ xue-feng@sgepri.sgcc.com.cn \\ * Correspondence: zhouxia@njupt.edu.cn; Tel.: +86-13-81-39-00-451
}

Citation: Zhou, X.; Lu, J.; Xie, X.; Bu, C.; Wan, L.; Xue, F. Communication Bandwidth Prediction Technology for Smart Power Distribution Business in Smart Parks. Electronics 2021, 10, 3143 https://doi.org/10.3390/

electronics10243143

Academic Editor: Maysam Abbod

Received: 3 November 2021

Accepted: 13 December 2021

Published: 17 December 2021

Publisher's Note: MDPI stays neutral with regard to jurisdictional claims in published maps and institutional affiliations.

Copyright: () 2021 by the authors. Licensee MDPI, Basel, Switzerland. This article is an open access article distributed under the terms and conditions of the Creative Commons Attribution (CC BY) license (https:// creativecommons.org/licenses/by/ $4.0 /)$.
Abstract: Accurate prediction of power business communication bandwidth is the premise for the effectiveness of power communication planning and the fundamental guarantee for regular operation of power businesses. To solve the problem of scientifically and reasonably allocating bandwidth resources in smart parks, communication bandwidth prediction technology of intelligent power distribution service for smart parks is proposed in this paper. First, the characteristics of mixed service data arrival rate of power distribution and communication mixed services in smart parks were analyzed. Poisson process and interrupted Poisson process were used to simulate periodic and sudden business of smart parks to realize accurate simulation of the business arrival process. Then, a service arrival rate model based on the Markov modulation Poisson process was constructed. An active buffer management mechanism was used to dynamically discard data packets according to the set threshold and achieve accurate simulation of the packet loss rate during the arrival of smart park business. At the same time, considering the communication service quality index and bandwidth resource utilization, a business communication bandwidth prediction model of smart parks was established to improve the accuracy of business bandwidth prediction. Finally, a smart power distribution room in a smart park was used as an application scenario to quantitatively analyze the relationship between the communication service quality and bandwidth configuration. According to the predicted bandwidth, the reliability and effectiveness of the proposed method were verified by comparison with the elastic coefficient method.

Keywords: smart park; communication bandwidth forecast; queuing theory; Markov modulated Poisson process; cache group management

\section{Introduction}

A variety of energy forms and types of smart terminals exist in smart parks [1-4]. The power business in smart parks is complex, such as distributed power business, energy consumption monitoring business, control business, etc., which demands higher requirements on edge computing and edge communication capabilities. Reasonable power communication planning is the basis for regular operation of power business in smart parks [5,6]. Problems such as redundancy and waste of communication network resources and the inability of communication service quality to meet the needs of communication services between terminals are caused by communication bandwidth planning that does not match the actual situation, thereby restricting the development of smart parks $[7,8]$. The rationality of power communication planning can be guaranteed by accurate power business bandwidth prediction $[9,10]$.

Research on the prediction of communication bandwidth of power distribution business has mainly focuses on certain aspects. The authors of [11] proposed a bandwidth calculation method based on $\mathrm{M} / \mathrm{M} / 1 / \mathrm{k}$ queuing theory for information collection services 
and allocated bandwidth resources with the help of the constraint relationship between communication service quality indicators and bandwidth utilization. However, this method does not consider the mixed data flow problem formed by the intelligent power distribution service and cannot solve the bandwidth prediction problem of mixed service. According to the difference between the arrival rate of mixed communication service and communication service quality, the authors of [12] predicted the bandwidth of cross service section by constructing a composite service convergence flow bandwidth prediction model. In addition, some experts have proposed the use of fourth-order hyperexponential distribution to simulate the arrival rate of mixed services [13]. Although this method can solve the simulation of the arrival process of mixed services, it simplifies the arrival rate of arriving data packets to Poisson distribution. The characteristics of different communication services in smart park are not considered in this method, and the specific analysis of the arrival of different types of service data is lacking, which is not suitable for the prediction of the communication bandwidth of power distribution service in smart park systems. In [14], a hybrid traffic model that includes self-similar traffic and Poisson traffic was proposed to predict the bandwidth of electricity consumption information collection services, but this method cannot meet the needs of complex communication services in smart park systems. With the improvement of computing power, the authors of [15] used intelligent algorithms to train bandwidth prediction models to achieve real-time bandwidth prediction for mobile internet services. However, the computational cost of this method is relatively high and it is difficult to popularize. The power service communication bandwidth prediction method based on intelligent algorithms, such as BP neural network, random forest algorithm, and ant colony optimization algorithm, can effectively improve the bandwidth prediction speed $[16,17]$. However, due to simplification of the construction process of the bandwidth prediction model, the bandwidth prediction is not as accurate. In engineering, it is nowadays common to refer to the guideline and adopt the method of combining visual observation and elastic coefficient to predict the bandwidth of the power distribution communication network, which is compatible with the traditional power distribution business [18]. However, the communication bandwidth result is usually more significant than the actual bandwidth demand, resulting in waste of communication resources and low bandwidth utilization. With changes in operational characteristics of smart park business, the abovementioned model cannot accurately describe the arrival process of smart park business data. It is difficult to achieve an accurate prediction of business bandwidth.

Given the above problems, this paper proposes a technology for predicting the communication bandwidth of smart power distribution services in smart parks. This method realizes the effective bandwidth prediction of service bandwidth with the goal of intelligent power distribution communication service quality requirement in smart parks. At the same time, starting from different business characteristics, it accurately simulates the data arrival process of smart power distribution and communication services in smart parks to improve the accuracy of bandwidth prediction.

First, according to the different characteristics of power communication services in smart parks, the arrival process of power communication data packets was simulated by interrupted Poisson process (IPP) and Poisson process to accurately describe the arrival process of communication mixed services data. Then, the characteristics of communication service convergence flow were analyzed, and the arrival rate of composite services was calculated. The active cache management mechanism was then used. Combined with the queuing theory model, the arrival of composite service data packets was modeled. Then, using the basic flow of power distribution service as the parameter, the bandwidth was predicted in combination with the real-time and reliability requirements of the service, and the bandwidth prediction model of intelligent power distribution communication service was established using the communication service quality index and bandwidth resource utilization. Finally, taking an intelligent distribution room in a smart park as an application scenario, the quantitative relationship between the communication service delay, packet loss rate, bandwidth utilization, and bandwidth configuration was analyzed, and the 
predicted bandwidth was obtained. The reliability and effectiveness of the proposed method were verified by comparison with the elastic coefficient method.

\section{Convergence Flow Characteristics of Communication Services in Smart Parks}

\subsection{Basic Characteristics of Smart Park Communication Services}

Smart parks adopt mature, reliable, economical, and suitable communication methods for business, such as $\mathrm{WiFi}$, EPON, industrial ethernet, 4G/5G wireless public network, etc. They support services such as intelligent identification and monitoring of each link of electricity distribution, electricity consumption information collection, distributed energy control, etc. to meet the data transmission needs between collection equipment, sensors, and smart terminals.

This section analyzes smart park services based on typical QoS indicators and characterizes the services. Smart power distribution services included in smart parks can be divided into four basic types: distributed power supply business, energy consumption monitoring business, energy control business, and video surveillance business. With regard to the QoS indicators of public network communication services and the "Technical Regulations for Power System Communication Design", the typical communication QoS indicators of the above four basic service categories are given in Table 1.

Table 1. Typical QoS indicators of communication services in smart parks.

\begin{tabular}{|c|c|c|c|c|}
\hline Business Type & Time Delay & Packet Loss Rate & Concurrency Rate & Way of Communication \\
\hline Distributed power supply business & $50 \mathrm{~ms}$ & $<0.1 \%$ & $20 \%$ & EPON, wireless private network \\
\hline Energy consumption monitoring business & second level & $<1 \%$ & $25 \%$ & LoRa, wireless private network \\
\hline Energy control business & $30 \mathrm{~ms}$ & $<0.01 \%$ & $100 \%$ & EPON, wireless private network \\
\hline Video surveillance business & second level & $<1 \%$ & $10 \%$ & Ethernet, wireless public network \\
\hline
\end{tabular}

According to the characteristics of communication services in smart parks, various services can be divided into periodic services and sudden services. Routine services are mostly expressed as typical applications of regular communication with machines. There are many types of routine business data, significant business traffic, and stable data generation, and most data transmission cycles are fixed values. Sudden services are primarily manifested as failures of monitoring points or abnormal control signals. This type of service has high requirements for real-time service and accuracy, and when mapped to QoS indicators, data delay and packet loss rate requirements are high. The two types of service data are independent of each other. The data enters the buffer queue at different arrival rates to form a mixed queue, and the aggregation node allocates bandwidth according to different service QoS indicators to meet the service requirements.

\subsection{Analysis of Convergence Flow Characteristics of Communication Services in Smart Parks}

The traditional network traffic model assumes that the arrival process of the data packet is a Poisson process. With the expansion of network scale and the continuous development of new power distribution services in smart parks, network traffic has shown strong burstiness and randomness. Therefore, it is difficult for the classic Poisson model to accurately simulate its characteristics.

In this work, the smart park business was divided into periodic business and sudden business. Periodic services include video multimedia communication services, such as smart meter collection, energy storage station monitoring, and on-site video equipment monitoring. Sudden services include data communication services, such as distributed power failure control, power distribution system failure information upload, and precise load shedding control services. Figure 1 shows the business convergence processing model for constructing business sections. 


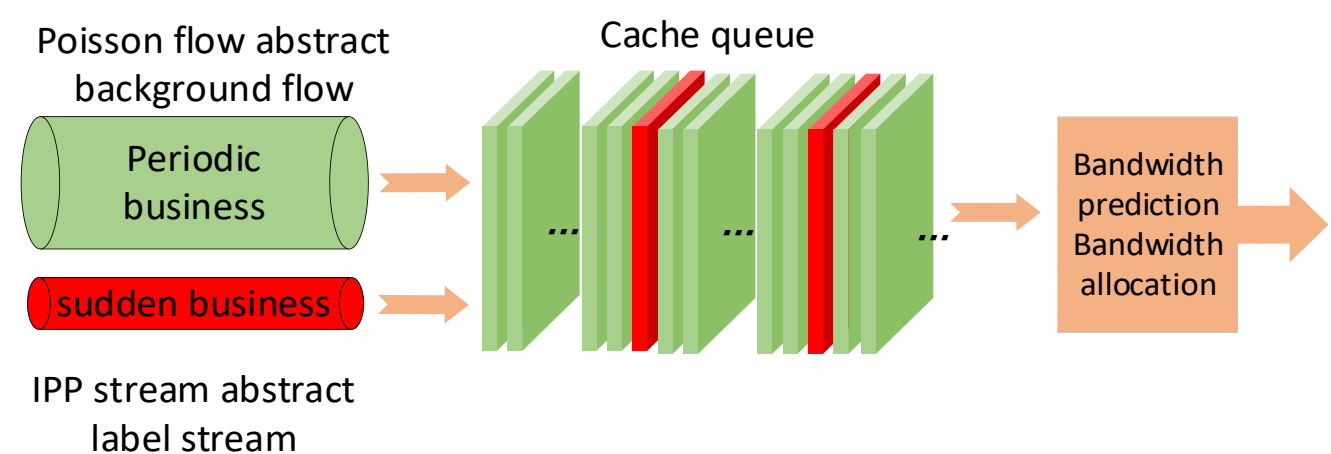

Figure 1. Convergence flow of intelligent power distribution and consumption communication service.

The Poisson process and the IPP were used to simulate the arrival process of periodic and burst business communication data packets, respectively. The system forwarding rate (bandwidth) in the service process was set to a fixed value. A mixed service queuing model was established to realize the analysis of the service convergence characteristics of smart parks. The details are as follows:

(1) Interrupted Poisson process: The Markov chain describing the IPP arrival process is shown in Figure 2, where $P_{a}$ represents the probability of staying in one state, and $P_{b}$ is the probability of staying in two states. When the IPP arrival process shifts to the two states, the arrival obeys the parameter $\lambda_{0}$ Poisson distribution.

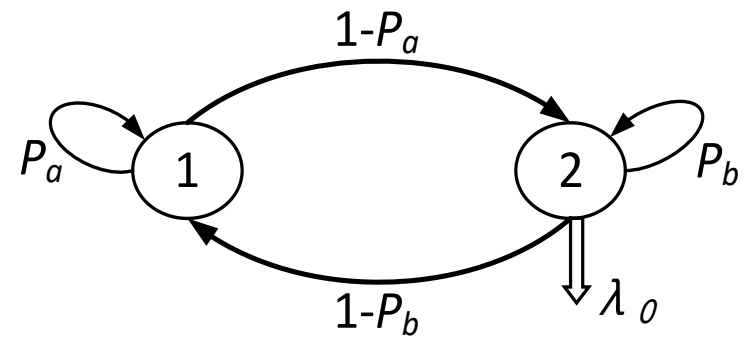

Figure 2. State transition diagram of an interrupted Poisson process.

(2) Poisson process: $\lambda_{1}$ represents the intensity of Poisson distribution arrival, which can be mapped to the arrival rate of periodic services.

The IPP stream and the Poisson stream were superimposed, which is equivalent to a Markov modulated Poisson process (MMPP) stream, and the complex mixed data stream was converted into a single arrival stream analysis [19] using the MMPP stream to describe the convergence flow of communication business data in smart parks. Compared with the previous use of traditional Poisson distribution to simulate the arrival rate of all service data, MMPP can more accurately simulate the arrival process of communication service data. It makes the bandwidth prediction model more realistic and improves the accuracy of bandwidth prediction under the premise of meeting the communication service quality.

\section{Communication Bandwidth Estimation Model and Solution Method Based on $\mathrm{MMPP} / \mathrm{m} / \mathrm{c} / n$ Queue}

3.1. Mixed Service Arrival Rate Model

The Poisson process with the parameter $\lambda_{1}$ and the interrupted Poisson process with the parameters $\lambda_{2}, p_{a}$, and $p_{b}$ can be superimposed and combined into a double random Poisson process, that is, a 2-state Markov modulated Poisson process (MMPP(2)). The corresponding parameters are $r_{1}, r_{2}, \lambda$, and $\lambda_{0}$, and the transfer formulas are as follows:

$$
\begin{gathered}
\mathrm{MMPP}(2) \rightarrow \mathrm{M}+\mathrm{IPP} \\
\lambda \rightarrow \lambda_{1}
\end{gathered}
$$




$$
\begin{gathered}
\lambda_{0} \rightarrow \lambda_{1}+\lambda_{2} \\
r_{1} \rightarrow\left(1-p_{a}\right), r_{2} \rightarrow\left(1-p_{\mathrm{b}}\right)
\end{gathered}
$$

In the formula, $\lambda$ represents the data packet arrival rate when $\mathrm{MMPP}(2)$ is in one state; $\lambda_{0}$ represents the data packet arrival rate when the MMPP(2) is in two states; $r_{1}$ represents the second-order Markov probability of the transition from state 1 to state two in the Poisson modulation process; $r_{2}$ represents the probability of transition from state two to state one in $\operatorname{MMPP}(2)$.

It can be seen that the infinitesimal generating matrix of the Markov chain in MMPP(2) is $Q$, and the strength matrix of the Poisson process is $R$. Then, the stable probability distribution $\Pi$ of the Markov chain is as follows:

$$
\begin{gathered}
Q=\left|\begin{array}{cc}
-r_{1} & r_{1} \\
r_{2} & -r_{2}
\end{array}\right|, R=\left|\begin{array}{cc}
\lambda_{1} & 0 \\
0 & \lambda_{2}
\end{array}\right| \\
\Pi=\left(\pi_{1}, \pi_{2}\right)=\frac{1}{r_{1}+r_{2}}\left(r_{2}, r_{1}\right)
\end{gathered}
$$

The average reaching rate of $\mathrm{MMPP}(2)$, that is, the arrival rate of mixed service data packets, is as follows:

$$
\bar{\lambda}=\frac{r_{2} \lambda_{1}+r_{1} \lambda_{0}}{\left(r_{1}+r_{2}\right)}=\frac{r_{2} \lambda_{1}+r_{1}\left(\lambda_{1}+\lambda_{2}\right)}{\left(r_{1}+r_{2}\right)}
$$

\subsection{Active Cache Management Mechanism}

In terms of packet loss rate, the random early detection (RED) algorithm manages packet loss by taking packet loss actions in advance. Setting the buffer threshold, when the number of data packets exceeds the threshold, data packets are randomly discarded to ensure communication efficiency. Still, the packet loss rate will gradually increase, as shown in Figure 3. The packet loss rate mainly affects QoS indicators. Considering the continuous improvement of QoS requirements for power communication services in smart parks, an active management mechanism for hybrid service aggregation node buffers is proposed to effectively reduce the packet loss rate and meet the QoS indicator requirements.

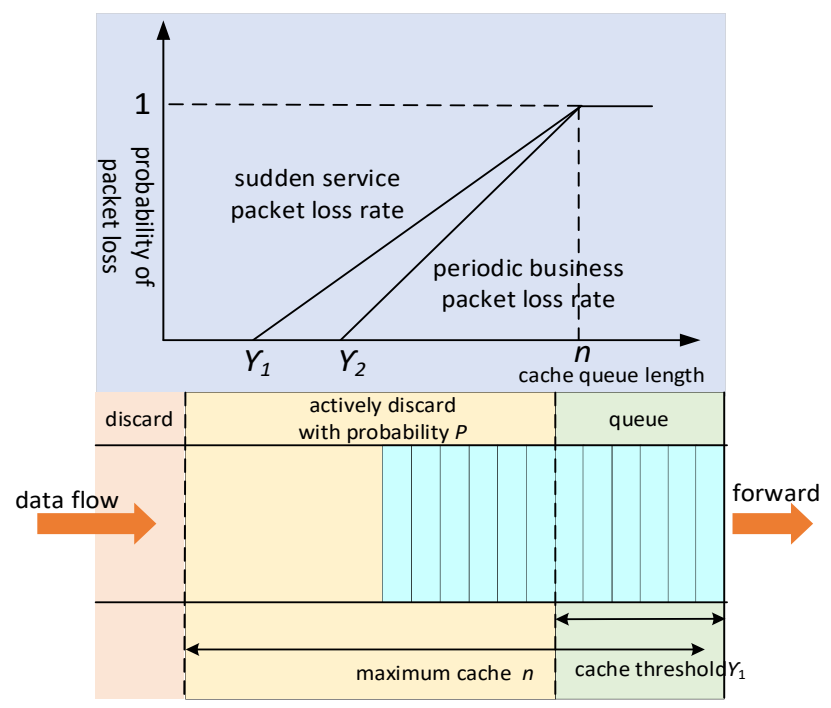

Figure 3. Active cache management mechanism of sink node for mixed services.

In Figure 3, a first-in-first-out (FIFO) single-server queuing system is shown. Two types of heterogeneous packets share the same buffer, and the maximum buffer packet length is $n$. The two types of packet data flow thresholds are represented by $\gamma_{1}$ and $\gamma_{2}$. The sudden business flow is simulated by the IPP, and the periodic business flow is affected by 
the traditional Poisson process. When the number of data packets in the system exceeds the set threshold $\gamma$, the incoming data packets will be queued in the data buffer for forwarding. When the number of data packets in the system exceeds the threshold but does not exceed the maximum packet buffer length $n$, the system will actively randomly discard the data packets with probability $\left(1-p_{i}\right)$, and packet loss will begin to occur, where $i(\gamma \leq i \leq n)$ represents the number of data packets in the system. When the number of data packets in the system exceeds the maximum packet buffer length $n$, the incoming data packets will not be queued and directly discarded.

\subsection{Performance Index Analysis and QoS Parameter Mapping Model}

Related studies have analyzed the $\mathrm{M} / \mathrm{m} / \mathrm{c}$ model and MMPP $/ \mathrm{m} / \mathrm{c}$ model with specific analytical expressions. The authors of [20] compared the average queue length and average waiting time of the two models by setting the same number of service desks and average arrival rate. It was found that when the MMPP contains fewer states, there is no noticeable difference in the queue length and waiting time between the two. Therefore, we can use the MMPP to accurately simulate the arrival process of mixed service packets and calculate the data packet arrival rate of the composite service flow. The mapping of packet loss rate and delay of communication services in the $\mathrm{MMPP} / \mathrm{m} / \mathrm{c}$ queuing model can be replaced by the calculation formulas of average queue length and average waiting time in the $\mathrm{M} / \mathrm{m} / \mathrm{c}$ queuing model, simplifying the calculation difficulty.

Relevant studies have shown that the $\mathrm{M} / \mathrm{m} / \mathrm{c}$ system has higher service efficiency and shorter queue length compared to multiple $\mathrm{M} / \mathrm{m} / 1$ systems. In this study, combined with the proposed active queue management mechanism, the RED algorithm was used to manage the packet loss problem. Multiple edge IoT gateways with the same forwarding rate were set up to forward the array packet queue, and a mixed service data queuing model was established. The $\mathrm{M} / m / c$ infinite buffer model was used to derive the $\mathrm{M} / \mathrm{m} / \mathrm{c} / \mathrm{n}$ performance index of the finite queue length according to Formulas (8)-(15):

The state transition probability formula is as follows:

$$
\begin{gathered}
P_{0}=\left[\sum_{k=0}^{c-1} \frac{(c \rho)^{k}}{k !}+\frac{(c \rho)^{c}}{c !} \frac{\left(1-\rho^{n-c+1}\right)}{1-\rho}\right]^{-1} \\
P_{k}= \begin{cases}\frac{c^{k} \rho^{k}}{k !} P_{0} & 0 \leq k<c \\
\frac{c^{c} \rho^{k}}{c !} P_{0} & c \leq k<n\end{cases}
\end{gathered}
$$

In $\rho=\frac{\lambda}{c \mu}$, the data packet rate of the mixed service flow is $\lambda$, which satisfies the MMPP distribution. The number of edge IoT gateways set in the queuing system is $c$. The forwarding rate of service data packets by a single edge IoT gateway is $\mu$, and the maximum value of the cache configuration node is $n$.

From this derivation, it can be seen that the buffer overflow probability of the $\mathrm{M} / \mathrm{M} / \mathrm{c} / n$ queue, that is, the data packet loss rate $P_{\text {loss }}$, is as follows:

$$
P_{\text {loss }}=\sum_{k=\gamma}^{n} p_{e} p_{k}=\sum_{k=\gamma}^{n} \frac{k}{n} \frac{c^{c} \rho^{k}}{c !} p_{0}
$$

In the formula, $\gamma$ represents the packet buffer threshold. When the number of data packets in the queue reaches the point, the system will actively discard the packets with the probability of $p_{e}$ and actively manage the data buffer.

Considering the packet loss rate, the actual arrival rate $\lambda e$ of packets is as follows:

$$
\lambda_{e}=\lambda\left(1-p_{\text {loss }}\right)
$$


The average data queue waiting for the captain $L s$ is as follows:

$$
L_{s}=L_{q}+c-\sum_{k=0}^{c-1}(c-k) p_{k}
$$

where $L_{q}$ is as follows:

$$
\begin{aligned}
& L_{q}=\sum_{k=c}^{n}(k-c) p_{k} \\
& =\frac{c^{c} \rho^{c+1} p_{0}}{c !(1-\rho)^{2}}\left[1-(n-c+1) \rho^{n-c}+(n-c) \rho^{n-c+1}\right]
\end{aligned}
$$

The average queuing time $W_{s}$ in the system and the delay $T_{S}$ is as follows:

$$
T_{s}=\frac{L_{s}}{\lambda_{e}}
$$

The system utilization rate $\eta$ is as follows:

$$
\eta=\frac{\lambda_{e}}{c \mu}
$$

\subsection{Solution of Bandwidth Prediction Model for Power Distribution Communication Service}

The $\mathrm{M} / \mathrm{M} / c / n$ queuing theory model is based on the constructed mixed service data flow, among the communication service QoS indicators. These parameters best reflect the communication quality of the intelligent power distribution service, including delay, packet loss rate, and bandwidth utilization. It can be seen from Formulas (10)-(15) that the packet delay $T_{S}$ of the mixed service, the composite service data loss rate $P_{\text {loss }}$, and the system bandwidth utilization rate $\eta$ are functions of the service data forwarding rate $\mu$.

In this study, energy monitoring services was taken as an example to construct a queuing theory smart park service communication bandwidth optimization model that takes the data loss rate and delays in the QoS indicators of the communication service as the constraint conditions and maximizes the bandwidth utilization, as the objective Function (16) shows:

$$
\left\{\begin{aligned}
\max _{\mu} \eta & =\frac{\lambda_{e}}{c \mu} \\
\text { s.t. } P_{\text {loss }} & \leq C_{\text {loss }} \\
T_{s} & \leq C_{T_{s}}
\end{aligned}\right.
$$

In the formula, $C_{\text {loss }}$ and $C_{T S}$ represent the minimum value of the packet loss rate and delay of the service types included in the hybrid service aggregation node.

Therefore, the implementation steps of the optimal bandwidth prediction algorithm for hybrid power communication services are as follows:

Step 1: Obtain the initial rate of arrival of various periodic service and sudden service packets using Formulas (1)-(4) to determine MMPP(2) state transition probability and use Formula (7) to calculate the mixed service achieved rate.

Step 2: Set the constraint conditions $S_{\text {loss }}$ of the periodic service and sudden service optimization models as the minimum packet loss rate of the service, set $S_{T s}$ as the minimum delay of the service, and determine the maximum grouping upper limit of the aggregation node buffer as $\left[S_{T s}^{*} n_{\max }\right]$.

Step 3: Set the value range and sampling interval of the predicted bandwidth and set the cache group variable $k=1$.

Step 4: Use the enumeration method to obtain the given cache grouping variable $(k=i)$ and use the nonlinear programming algorithm to obtain the bandwidth utilization ratio $\eta_{i}$ of the periodic service and sudden service under the condition of $S_{\text {loss }}$ and $S_{T s}$ as the constraints of the corresponding forwarding rate of the predicted bandwidth $\mu_{i}$. 
Step 5: Set the bandwidth utilization rate $\eta i$ of the periodic service and the sudden service to the bandwidth utilization rate of the sink node when the corresponding cache packet is $i$ and set the corresponding sink node forwarding rate, that is, the expected bandwidth $\mu_{i}$ to the predicted bandwidth.

Step 6: Update the sink node cache group variable $k=k+1$. If $k$ is less than the sink node cache maximum packet upper limit $\left[S_{T_{s}}{ }^{*} n_{\max }\right]$, go to step 4; otherwise, go to step 7 .

Step 7: Calculate the optimal solution of the model $\eta=\max \{\eta i)$ and its corresponding forwarding rate $\mu_{i}$ of the sink node, which is recorded as the primary predicted bandwidth value of the service section.

\section{Analysis of Calculation Examples of Communication Service Bandwidth Prediction}

First of all, the quantitative relationship between the communication service quality index and the predicted bandwidth was analyzed based on the queuing theory for power communication business in smart parks to verify the reliability of the bandwidth prediction model proposed in this article. Then, using the smart power distribution room in smart parks as an application scenario, based on relevant data, the communication service bandwidth was predicted and compared with the traditional elasticity coefficient method to verify the superiority and reliability of the smart power distribution communication bandwidth prediction method proposed in this paper.

\subsection{Reliability Experiment and Analysis of Bandwidth Prediction Model}

Using the complete enumeration method to find the buffered packets $(n=i)$, the relationship between QoS index parameters, such as data delay, packet loss rate, bandwidth utilization, etc., and the forwarding rate was analyzed, as shown in Figures 4-6. The dynamic link between QoS index parameters and bandwidth prediction was analyzed. For the convenience of observation, the bandwidth utilization corresponding to the bandwidth configuration of the node that cannot meet the communication QoS index was set to 0 , as shown in Figures 4-6.

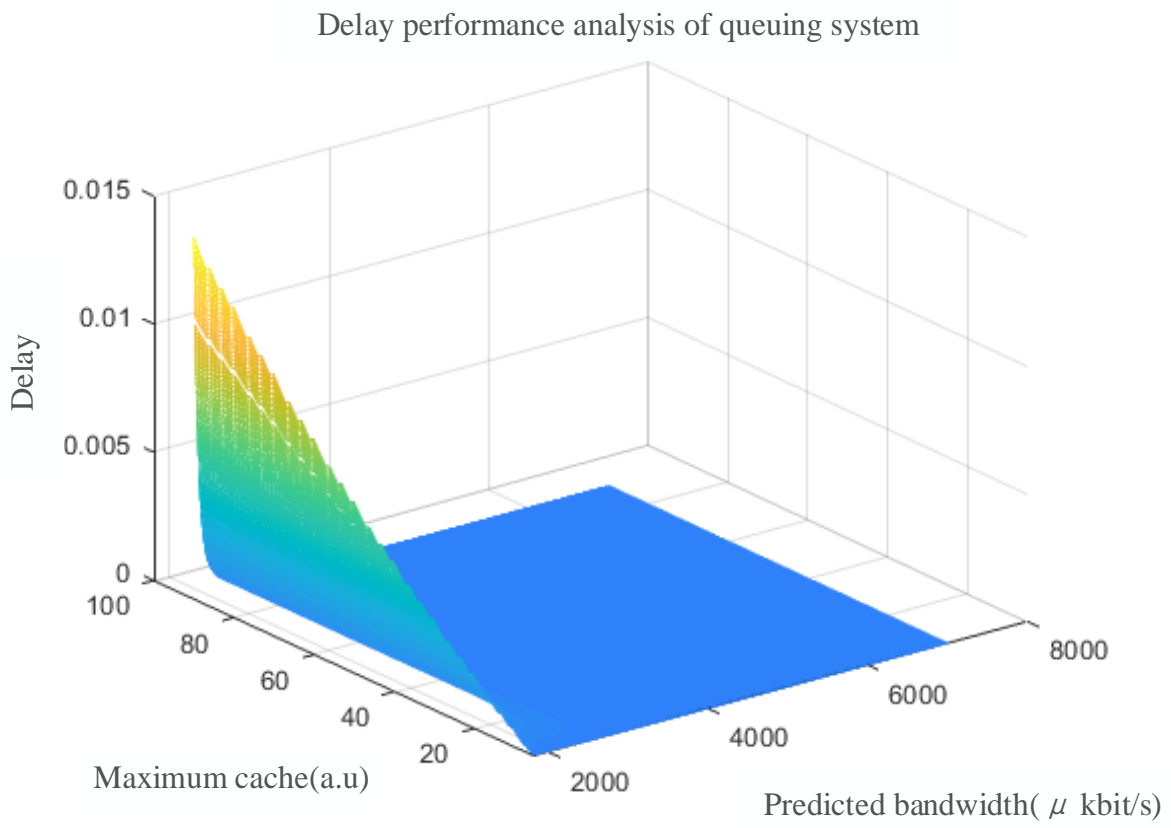

Figure 4. Delay performance analysis of queuing system. 


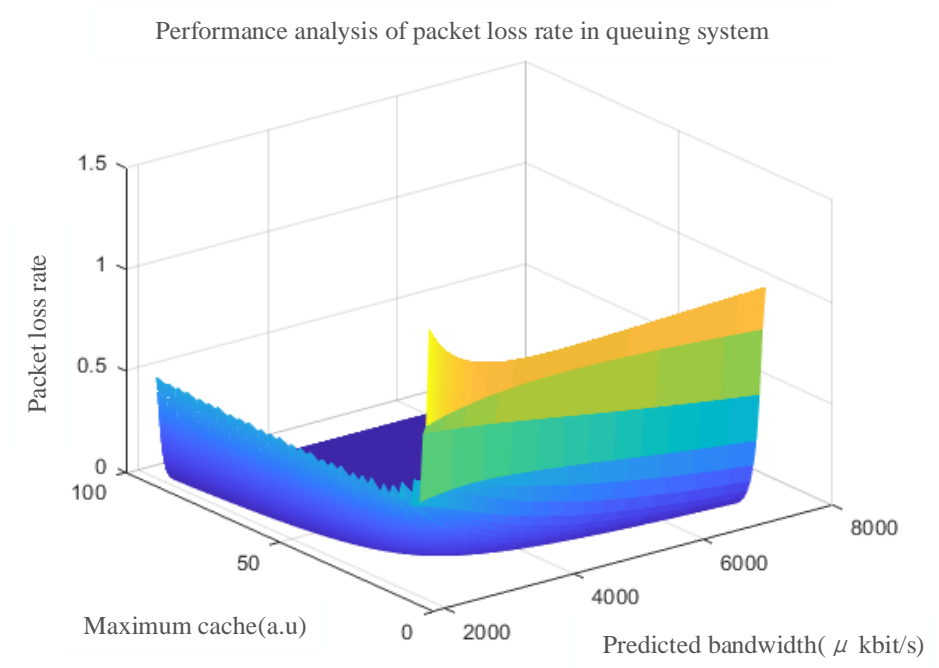

Figure 5. Performance analysis of packet loss rate in the queuing system.

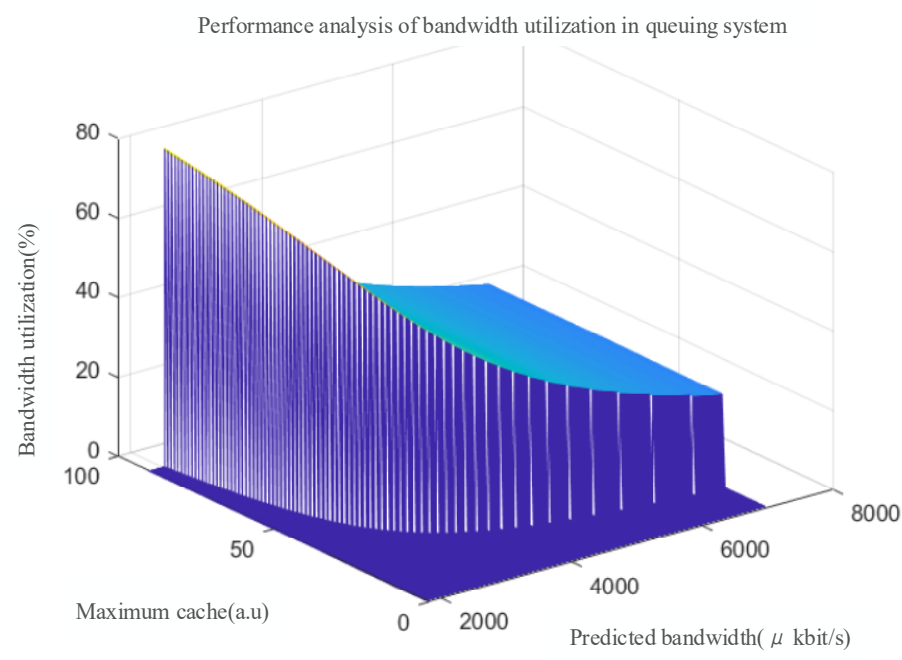

Figure 6. Performance analysis of bandwidth utilization in the queuing system.

From the analysis in Figure 4, it can be seen that when the node bandwidth is configured as a fixed value, the larger the number of system cache queues and the higher the system data delay. When the number of cache queues in the system is a fixed value, the larger the node bandwidth configuration and the lower the data delay. At the same time, as the number of node caches continues to increase, the impact of node bandwidth on system latency becomes more apparent.

It can be seen from the analysis in Figure 5 that when the system buffer packet length remains the same, the system data packet loss rate keeps decreasing as the node bandwidth configuration increases. When the node bandwidth is configured as a fixed value, the system buffer packet length continues to increase, and the system data packet loss rate continues to decrease. At the same time, when the system buffer packet length is low, the node bandwidth configuration has a more noticeable impact on the system data packet loss rate.

It can be seen from the analysis in Figure 6 that when the system cache packet length is a fixed value, the node bandwidth utilization rate is inversely proportional to the node bandwidth configuration. When the node bandwidth is configured as a fixed value, the change of the system buffer packet length has little effect on the system bandwidth utilization.

From the above analysis, the following observations can be made: (1) If you need to meet the communication QoS indicators of each business, you need to configure a certain system buffer packet length and node bandwidth. (2) When the system buffer packet 
length is reasonably configured, with the increase in node communication bandwidth, the communication QoS performance, such as system delay and system packet loss rate, will continue to improve and the service quality of power communication services will be enhanced. However, an over-configured node bandwidth will affect the system bandwidth utilization and cause waste of communication resources. (3) When a specific node bandwidth is configured, with the continuous increase in the system buffer packet length, the system packet loss rate gradually decreases, but the system delay will increase. This means that an appropriate increase in the system buffer packet length will improve the accuracy performance (packet loss rate) of the power communication service but will sacrifice part of the real-time performance (delay).

\subsection{Example Analysis of Bandwidth Prediction Optimization Model}

To verify the bandwidth prediction model for smart power distribution and communication services proposed in this article, a smart power distribution room in a smart park demonstration project was selected as an application scenario. Various data types, such as environmental monitoring, equipment status, and energy consumption information collection, were connected to different communication interfaces through other communication methods, and the edge IoT gateway was entered to queue for forwarding. At the same time, parameters such as the basic flow of power communication services in the smart power distribution room and the number of smart terminals were given, the bandwidth required for communication services of the smart power distribution room in the smart park was analyzed, and the predicted bandwidth was obtained. According to the type of intelligent terminal configuration of the power distribution room, the power distribution room was divided into four types, which were marked as (1)-(4) and used to verify the relationship between the predicted bandwidth value and the size of the communication service traffic in the power distribution room. Video monitoring equipment was connected to the edge IoT gateway by optical fiber, and the rest of the device data was connected to the edge IoT gateway by WiFi, LoRa, bus, 4G/5G wireless public network, and the services included in various types of power distribution rooms of the distribution network. The detailed configuration table of the types and smart terminal equipment is shown in Table 2.

Table 2. The actual configuration of four types of intelligent distribution rooms in a smart park.

\begin{tabular}{|c|c|c|c|c|c|c|c|}
\hline \multirow[t]{2}{*}{ Business Type } & \multirow[t]{2}{*}{ Equipment Type } & \multicolumn{2}{|c|}{$\begin{array}{l}\text { Basic Business } \\
\text { Flow(kbit/s) }\end{array}$} & \multicolumn{4}{|c|}{$\begin{array}{l}\text { Configuration Quantity of Each Type of } \\
\text { Power Distribution Room/Piece }\end{array}$} \\
\hline & & $\begin{array}{l}\text { Sudden } \\
\text { services }\end{array}$ & $\begin{array}{l}\text { periodic } \\
\text { service }\end{array}$ & (1) & (2) & (3) & (4) \\
\hline \multirow{4}{*}{$\begin{array}{l}\text { Distributed power } \\
\text { supply business }\end{array}$} & Distributed power control equipment & 12 & 80 & 2 & 3 & 1 & 1 \\
\hline & Distributed power supply access metering equipment & 20 & 90 & 1 & 2 & 2 & 2 \\
\hline & Power quality testing equipment & 15 & 50 & 2 & 3 & 2 & 2 \\
\hline & Smart meter & 1 & 3 & 450 & 300 & 250 & 300 \\
\hline Energy consumption & Environmental monitoring equipment & 20 & 80 & 2 & 1 & 2 & 3 \\
\hline \multirow{2}{*}{ monitoring business } & Energy storage station monitoring equipment & 30 & 60 & 2 & 1 & 3 & 2 \\
\hline & Equipment operating condition monitoring equipment & 25 & 120 & 2 & 1 & 2 & 2 \\
\hline \multirow{2}{*}{ Energy control business } & Distribution automation equipment & 12 & 60 & 1 & 2 & 1 & 2 \\
\hline & Electricity load demand side response equipment & 15 & 50 & 1 & 2 & 2 & 3 \\
\hline Video surveillance & Energy storage field video monitoring equipment & 200 & 1800 & 1 & 1 & 2 & 3 \\
\hline business & Intelligent building monitoring equipment & 300 & 1500 & 1 & 1 & 2 & 2 \\
\hline
\end{tabular}

The main parameter settings of the experiment were as follows: (1) The number of edge IoT gateways in the four types of power distribution rooms was set to 4 , that is, the value of $c$ in the queuing system was 4. (2) The maximum number of cached data packets $k$ at the edge IoT gateway was taken as $\lambda \bullet C_{T S}$ (rounded down). The data packet size was taken as 512 bytes. (3) The communication service quality index QoS was taken as the most stringent condition in the intelligent power distribution business, in which $C_{T s}$ was taken as 0.03 and $C_{\text {loss }}$ was taken as $0.1 \%$.

According to the intelligent distribution and utilization communication service bandwidth prediction method proposed in this article, using the corresponding service flow of various services and the arrival rate of sudden services and periodic services, the arrival rate of the mixed service was calculated using Formula (7), and the arrival rate of the composite service was calculated as a composite service packet. The edge IoT gateway 
rate is shown in Table 3. According to Formulas (10)-(15), four types of intelligent power distribution rooms were used to predict the relationship between the communication bandwidth and its network performance (bandwidth utilization, delay, and packet loss rate), as shown in Figures 7-9. The curve segment where the bandwidth utilization rate is 0 indicates that the predicted communication bandwidth failed to meet the delay and packet loss rate requirements.

Table 3. The arrival rate of communication mixed services in the intelligent power distribution room.

\begin{tabular}{cccc}
\hline $\begin{array}{c}\text { Power Distribution } \\
\text { Room Type }\end{array}$ & $\begin{array}{c}\text { Sudden Service } \\
\text { Arrival Rate }\end{array}$ & $\begin{array}{c}\text { Periodic Service } \\
\text { Arrival Rate }\end{array}$ & $\begin{array}{c}\text { Mixed Service } \\
\text { Packet Arrival Rate }\end{array}$ \\
\hline (1) & $1.17 \mathrm{Mbit} / \mathrm{s}$ & $6.74 \mathrm{Mbit} / \mathrm{s}$ & $7.09 \mathrm{Mbit} / \mathrm{s}$ \\
$(2)$ & $1.03 \mathrm{Mbit} / \mathrm{s}$ & $5.13 \mathrm{Mbit} / \mathrm{s}$ & $5.40 \mathrm{Mbit} / \mathrm{s}$ \\
$(3)$ & $1.95 \mathrm{Mbit} / \mathrm{s}$ & $8.25 \mathrm{Mbit} / \mathrm{s}$ & $4.43 \mathrm{Mbit} / \mathrm{s}$ \\
(4) & $1.76 \mathrm{Mbit} / \mathrm{s}$ & $10.28 \mathrm{Mbit} / \mathrm{s}$ & $1.17 \mathrm{Mbit} / \mathrm{s}$ \\
\hline
\end{tabular}

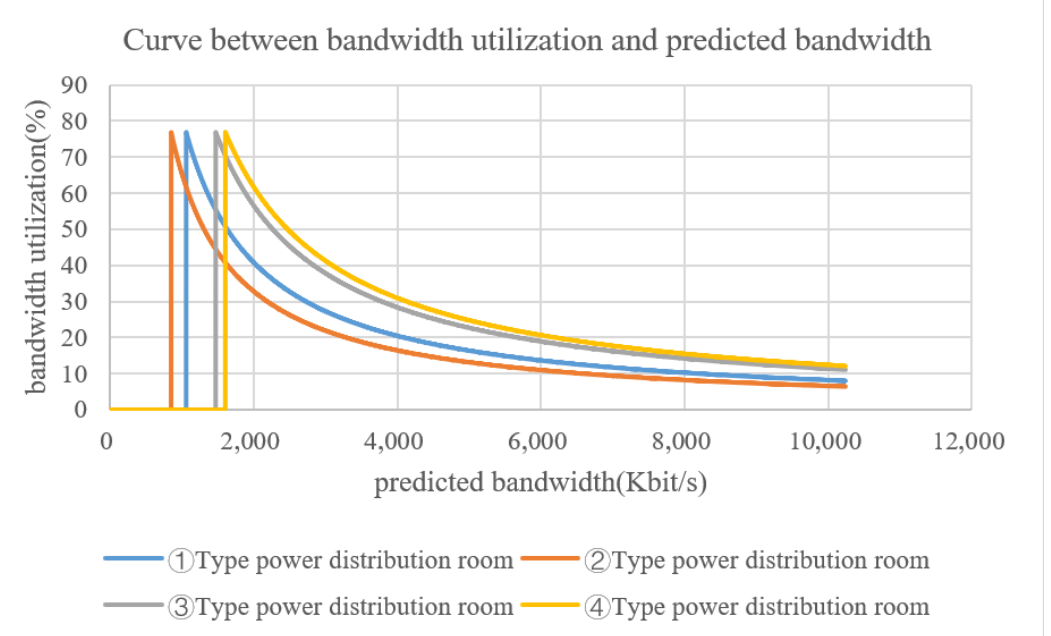

Figure 7. Curve between bandwidth utilization and predicted bandwidth.

Curve between packet loss rate and predicted bandwidth

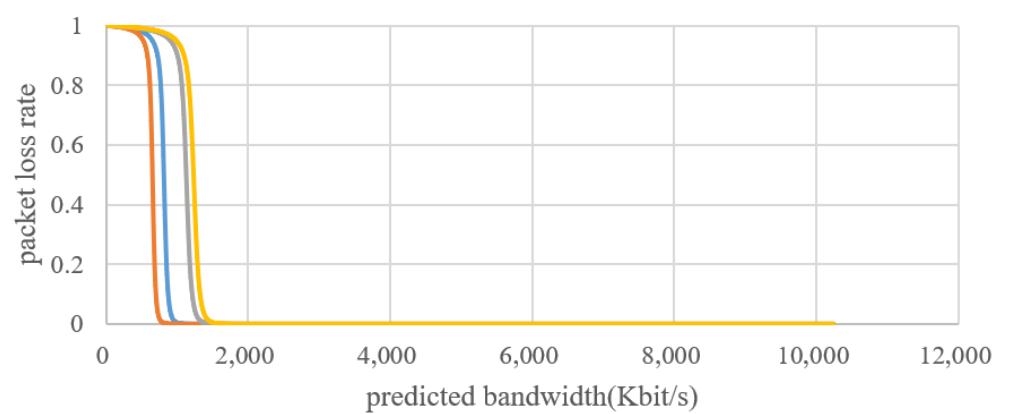

- (1)Type power distribution room - (2) Type power distribution room

Figure 8. Curve between packet loss rate and predicted bandwidth. 


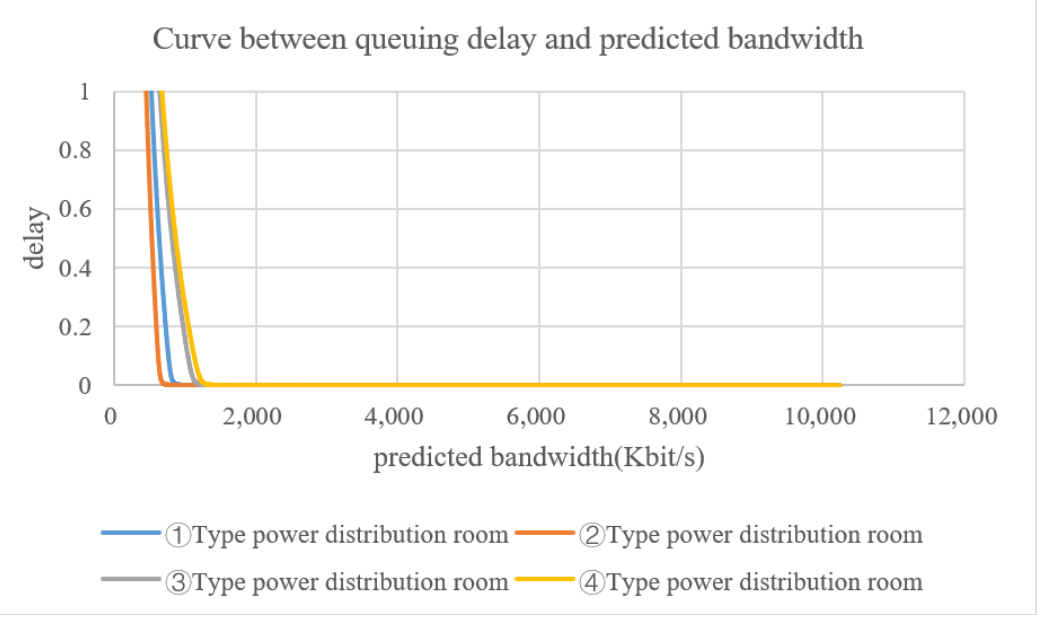

Figure 9. Curve between queuing delay and predicted bandwidth.

From the figure, the following observations can be made:

(1) On the premise of meeting the basic requirements of mixed service data transmission bandwidth, as the node bandwidth gradually increases, the network delay and packet loss rate decrease progressively, and the system utilization rate also gradually decreases. When the demand for power distribution and communication services in the smart park is great and the QoS requirements are high, the QoS indicators of the service can be improved by appropriately increasing the bandwidth configuration of the aggregation node to ensure normal and safe operation of the power distribution and communication services in the smart park. However, blindly increasing the bandwidth will lead to a decrease in bandwidth utilization, resulting in a waste of communication resources.

(2) The predicted bandwidth of different types of power distribution rooms in the smart park is related to the actual configuration of the power distribution room. The higher the power distribution room (the greater the amount of communication service access), the greater the predicted bandwidth value. When the bandwidth is configured below the critical bandwidth, the communication service quality indicators of the communication services accessed in the distribution network will not be met.

(3) As shown in Figure 8, with the continuous improvement of bandwidth configuration, the packet loss rate first drops slowly and then decreases rapidly, which is caused by taking packet loss actions in advance to manage the packet loss problem. The optimized packet loss rate calculation method is more suitable for processing data packets in actual routing and improves the accuracy of bandwidth prediction.

Table 4 shows the predicted bandwidth of the business section of an intelligent power distribution room. In the table, the maximum traffic bandwidth of the mixed business through the power distribution room business section is business traffic $\times$ concurrent ratio: $0.41 \times 100 \%+2.41 \times 20 \%+0.13 \times 25 \%+3.71 \times 10 \%=1.3 \mathrm{Mbit} / \mathrm{s}$.

Table 4. Calculation table of communication service bandwidth in the intelligent distribution room.

\begin{tabular}{|c|c|c|c|c|c|}
\hline Business Type & $\begin{array}{c}\text { Business } \\
\text { Flow/Mbit/s }\end{array}$ & $\begin{array}{c}\text { Concurrency } \\
\text { Ratio }\end{array}$ & $\begin{array}{l}\text { Redundancy } \\
\text { Factor }\end{array}$ & Delay/s & $\begin{array}{c}\text { Packet Loss } \\
\text { Rate/\% }\end{array}$ \\
\hline Power distribution operation control business & 0.41 & $100 \%$ & 2 & $\leq 0.1$ & $\leq 0.01$ \\
\hline Electricity distribution information collection business & 2.41 & $20 \%$ & 1 & $\leq 2$ & $\leq 1$ \\
\hline Distributed power business & 0.13 & $25 \%$ & 1.5 & $\leq 1$ & $\leq 5$ \\
\hline Video surveillance business & 3.71 & $10 \%$ & 1 & $\leq 3$ & $\leq 2$ \\
\hline
\end{tabular}

At present, the bandwidth prediction technology commonly used in engineering is the elastic coefficient method, which is determined by the redundancy coefficient and the disaster tolerance coefficient, and its maximum theoretical bandwidth utilization 
rate is about $66.6 \%$. This method can meet the requirements of power distribution and communication services, and the predicted bandwidth is about $1.95 \mathrm{Mbit} / \mathrm{s}$.

For the analysis in Table 5, a particular power distribution room in the smart park was selected and the elastic coefficient method commonly used in bandwidth prediction and the bandwidth prediction method proposed in this article was used to compare the calculated bandwidth prediction value with QoS indicators. On the premise of meeting the communication service quality requirements (delay $\leq 30 \mathrm{~ms}$ and packet loss rate $\leq 0.1 \%$ ), the predicted bandwidth calculated by the method proposed in this article was $1.56 \mathrm{Mbit} / \mathrm{s}$ and the bandwidth utilization rate was $76.93 \%$.

Table 5. Comparison of the predicted value of communication service bandwidth in intelligent distribution room.

\begin{tabular}{ccccc}
\hline Method & Bandwidth Prediction/(Mbit/s) & Bandwidth Utilization/\% & Delay/s & Packet Loss Rate/\% \\
\hline Traditional method & 1.95 & $66.6 \%$ & satisfy \\
Method of this paper & 1.56 & $76.93 \%$ & 0.03 & $0.1 \%$ \\
\hline
\end{tabular}

From Table 5, the following observations can be derived:

(1) Compared with the theoretical maximum bandwidth utilization rate of $66.6 \%$ of the traditional method, the bandwidth utilization rate of the method proposed in this paper was $76.93 \%$, which is an increase of more than $10 \%$. The communication service packet loss rate and delay were $0.1 \%$ and $0.03 \mathrm{~s}$, respectively, which are lower than the most stringent communication service quality indicators in the communication service section and meet the communication needs of the power distribution service in smart parks.

(2) Traditional methods are limited to qualitative analysis that meets the QoS indicators of communication services, and the bandwidth utilization rate is not high, which is likely to cause computational waste. In the context of smart parks, massive power distribution and communication business requirements, more stringent QoS index conditions, and the adoption of the elastic coefficient method will result in more significant waste of resources.

\section{Conclusions}

(1) In this study, the arrival process of periodic services and sudden services were simulated based on IPP flow and Poisson flow, which is more in line with the reality of smart power distribution and communication services in smart parks. The use of MMPP(2) to analyze the rate of appearance of mixed services can achieve accurate simulation of the rate of arrival of services in smart parks. It can be seen from the calculation example that, compared with the widely used elastic coefficient bandwidth prediction method, the utilization rate of the communication bandwidth is improved under the premise of ensuring the quality of communication service.

(2) The method proposed in this paper can realize accurate simulation of the packet loss process of business data of smart parks. The change trend of the packet loss rate shown in Figure 8 shows that the introduction of the active buffer management mechanism can dynamically analyze the packet loss rate of communication business data. This improves the accuracy of the bandwidth prediction model of the smart campus system.

(3) The smart park service communication bandwidth prediction model constructed in this work can effectively simulate the arrival process, reaching rate, and packet loss rate of different services in smart parks. It meets the characteristics of complex power distribution and communication services and high communication quality requirements in the smart park system, effectively improves the efficiency of service bandwidth prediction, and has important engineering practical value.

In smart parks, random services and periodic services have different application requirements, so a priority forwarding mechanism is required to ensure real-time services. In the method proposed in this paper, the impact of random service priority nature on bandwidth prediction is not considered. Therefore, the priority queue model for intelligent power distribution and utilization business of the smart park system will be the next research direction. 
Author Contributions: Conceptualization, X.Z. and J.L.; methodology, X.X. and J.L.; software, X.Z. and C.B.; validation, J.L., X.Z. and L.W.; formal analysis, X.Z., F.X.; investigation, L.W. and X.X.; resources, X.X., F.X.; data curation, J.L.; writing-original draft preparation, X.Z. and J.L.; writing-review and editing, X.Z. and X.X.; visualization, J.L., F.X.; supervision, X.Z., F.X.; funding acquisition, X.Z. All authors have read and agreed to the published version of the manuscript.

Funding: This research was funded by the National Natural Science Foundation of China, grant number 62022044; the Key Program of National Natural Science Foundation of China, grant number 61933005; and the Natural Science Foundation of Nanjing University of Posts and Telecommunications, grant number NY219094.

Data Availability Statement: Data is contained within the article.

Conflicts of Interest: The authors declare no conflict of interest.

\section{References}

1. Quelhas, A.; Gil, E.; Mccalley, J.D.; Ryan, S.M. A multiperiod generalized network flow model of the U.S. integrated energy system: Part I-model description. IEEE Trans. Power Syst. 2007, 22, 829-836. [CrossRef]

2. Colak, I.; Sagiroglu, S.; Fulli, G.; Yesilbudak, M. A survey on the critical issues in smart grid technologies. Renew. Sustain. Energy Rev. 2016, 54, 396-405. [CrossRef]

3. Quelhas, A.; McAlley, J.D. A Multiperiod Generalized Network Flow Model of the U.S. Integrated Energy System: Part II-Simulation Results. IEEE Trans. Power Syst. 2007, 22, 837-844. [CrossRef]

4. Giannopoulos, G.; Galbusera, L.; Theodoridis, G. Intelligent Energy Systems: Introducing Power-ICT Interdependency in Modeling and Control Design. IEEE Trans. Ind. Electron. 2015, 62, 2468-2477.

5. Bedi, G.; Venayagamoorthy, G.K.; Singh, R.; Brooks, R.; Wang, K.C. Review of internet of things (iot) in electric power and energy systems. IEEE Internet Things J. 2018, 5, 847-870. [CrossRef]

6. Bahram, S.; Amjad, A.M.; Juan, V.; Josep, G. Internet of things for modern energy systems: State-of-the-art, challenges, and open issues. Energies 2018, 11, 1252.

7. Huang, J. OPNET Modeler Manages Utilization, Guarantees Quality of Service for Wireless Networks. IEEE Commun. Mag. 2000, 1, 12-22.

8. Wu, J.; Yang, C.; Chen, B. Proactive Caching and Bandwidth Allocation in Heterogenous Networks by Learning from Historical Numbers of Requests. IEEE Trans. Commun. 2020, 68, 4394-4410. [CrossRef]

9. $\mathrm{Wu}, \mathrm{G}$. Application of adaptive resource allocation algorithm and communication network security in improving educational video transmission quality. Alex. Eng. J. 2021, 60, 4231-4241.

10. Wang, Y.J.; Shao-Cong, L.I.; Zhang, L. A New Method of Traffic Estimation and Bandwidth Planning of Power Grid Transmission Network. Telecommun. Electr. Power Syst. 2012, 33, 35-39.

11. Xu, Z.; Lu, J.; Li, Z.; Zhu, Y.P. Queue-Theory-Based Service-Section Communication Bandwidth Calculation for Power Distribution and Utilization of Smart Grid. In Proceedings of the 2015 8th International Conference on Intelligent Networks and Intelligent Systems (ICINIS), Tianjin, China, 1-3 November 2015; pp. 137-140.

12. Lu, J.; Li, Z.; Zhu, Y.P.; Xu, Z.Q. Communication bandwidth prediction for information gathering services in power distribution and utilization of smart grid. Power Syst. Technol. 2016, 40, 1277-1282.

13. Xu, Z.Q.; Lu, J.; Zhai, F.; Chuang, Z.; Gangjun, G. Communication Bandwidth Prediction of Aggregation of Multi-Services for Smart Power Distribution and Utilization. Power Syst. Technol. 2015, 39, 712-716.

14. Chen, S.D.; Chen, X.Q.; Huang, R.; Lu, J.; Wang, K.L. Bandwidth Prediction Method for Power Consumption Information Collecting Service Based on Mixed-Flow Model. Electr. Power Inf. Commun. Technol. 2018, 16, 39-44.

15. Mei, L.F.; Hu, R.C.; Cao, H.W.; Liu, Y.; Han, Z.; Li, F.; Li, J. Realtime mobile bandwidth prediction using LSTM neural network and Bayesian fusion. Comput. Netw. 2020, 182, 107515. [CrossRef]

16. Ruan, L.; Dias, M.; Wong, E. Machine Learning-Based Bandwidth Prediction for Low-Latency H2M Applications. IEEE Internet Things J. 2019, 6, 3743-3752. [CrossRef]

17. Ruan, L.; Mondal, S.; Wong, E. Machine Learning Based Bandwidth Prediction in Tactile Heterogeneous Access Networks. In Proceedings of the IEEE Conference on Computer Communications Workshops (INFOCOM WKSHPS), Honolulu, HI, USA, 15-19 April 2018; pp. 1-2.

18. Liu, X.J.; Zhang, C.; Liu, Z.Y.; Lu, J. A novel qos bandwidth prediction method for power distribution and utilization grid. Appl. Mech. Mater. 2015, 743, 551-554. [CrossRef]

19. Geleji, G.; Perros, H. Jitter analysis of an IPP tagged traffic stream in an $\{\mathrm{IPP}, \mathrm{M}\} / \mathrm{M} / 1$ queue. Ann. Telecommun. 2014, 69, 283-294. [CrossRef]

20. Jia, Y.; Zhang, Z.G.; Xu, T. Improving the Performance of MMPP/M/C Queue by Convex Optimization-A Real-World Application in Iron and Steel Industry. IEEE Access 2020, 8, 185909-185918. [CrossRef] 